\title{
Varus stress for easy visualization of the posterior horn of medial meniscus: an arthroscopy technical note
}

\begin{abstract}
Background: The posterior horn of medial meniscus is one of the most common sites of meniscal injuries, which can be difficult to access in most knees.

Methods: We describe an important maneuver for adequate visualization of the posterior horn of medial meniscus through the anterolateral portal during knee arthroscopy, by applying varus stress on a 90 degrees flexed knee.
\end{abstract}

Results: This technique opens out the posteromedial compartment of the knee to adequately visualize the medial meniscus posterior horn lesions and treat them.

Conclusion: Using this technique, surgeons avoid struggling with portals and instruments, therefore, minimizing articular surface damage, and refrain from unnecessary excessive valgus stress, therefore, preventing injury of the medial collateral ligament.

Level of Evidence: V

Keywords: Knee arthroscopy, Arthroscopic meniscectomy, Posterior horn, Medial meniscus, Varus stress, Injury, Surgeons, Portals, Instruments
Volume 6 Issue 4 - 2016

\author{
Manaf HYounis, Mazhar Fuad, Saleh Al- \\ Yazedi \\ Orthopedic Surgery Department, Qatar
}

Correspondence: Manaf H Younis, Department of Orthopedic Surgery, Hamad Medical Corporation, PO. Box 3050, Doha, Qatar Email myounis I@hamad.qa

Received: October 19, 2014 | Published: November II, 2016

\section{Abbreviations}

MCL, Medial Collateral Ligament, ACL, Anterior Cruciate Ligament, PCL, Posterior Cruciate Ligament

\section{Introduction}

The posterior horn of medial meniscus is the most common site of meniscal injury ${ }^{1}$ Knee meniscus has many vital roles in promoting the function of the knee; including but not limited to distributing and absorbing loads, joint proprioception and stability. It is well-proven that the medial meniscus is less mobile than the lateral one; partly due to its steady attachment to the Medial Collateral Ligament (MCL), and in particular, its posterior horn is firmly fixed to the posterior inter-condyloid fossa of the tibia, known as "Meniscal root". On top of that, the medial meniscus bears more load of the body weight, with consequent wear and tear, therefore, rendering it more susceptible to injury than its lateral counterpart. ${ }^{2}$

Understanding the biomechanical function of the meniscus in conserving the knee joint and preventing premature degenerative changes, has led to radical refinements in meniscal surgeries. ${ }^{3}$ shifting the trends from total meniscectomy into meniscal repair or minimal partial meniscectomy in case of irreparable meniscus tear. ${ }^{1,2,4}$ Proper visualization of the whole meniscus is imperative in making the best possible surgical decision. ${ }^{5,6}$ Unfortunately, the posterior horn of medial meniscus and its root can be difficult to visualize in most knees; partly due to the far posterior position compared to its counterpart, in addition to the convex larger medial femoral condyle.

The conventional method of valgus stress may not facilitate full posterior visualization and instrumentation without Medial Collateral Ligament (MCL) injury or damaging intra-articular cartilage. Few new techniques and maneuvers have been presented to facilitate the access to the posteromedial corner using new portals and instruments. ${ }^{7-10}$ or by doing soft tissue release. ${ }^{11}$
Here, we present an easy technique of varus positioning of the knee during arthroscopy, in order to promote the best visualization of the whole posterior horn of medial meniscus and allow instrumentation for resection/ repair without causing articular scuffing or using extra portals and instruments.

\section{Materials and methods}

\section{Technique}

On a standard operating table, and a regular lateral support, the side of the bed is well padded (Figure 1), prepping and draping, a tourniquet is applied and inflated, and standard anteromedial and anterolateral portals are inserted in the knee.

The knee is examined in the systematic routine; Suprapatellar pouch, lateral and medial gutters, patella undersurface are inspected in the knee-extended position. Then patellar tracking is assessed. Afterwards, the intercondylar notch with the Anterior Cruciate Ligament (ACL) and Posterior Cruciate Ligament (PCL) and the anterior horns of meniscus are examined with the knee in 90 degrees of flexion.

The medial compartment is examined as the knee is progressively brought into valgus and extension. The posterior horn of medial meniscus can be difficult to see, for which only 20-30 degrees of flexion and external rotation would help. Even then, bringing in the instruments for partial resection or meniscal suturing can be technically challenging and might damage the articular cartilage, or even injure the medial collateral ligament, if excessive valgus stress is applied.

\section{Varus positioning of the knee to visualize the posterior horn and root of medial meniscus}

The knee is brought to the side of the well-padded operating table and flexed up to 90 degrees. Thereafter, varus stress is applied on 
the leg by the operating surgeon (Figure 1-3). With the scope in the intercondylar notch just medial to the Anterior Cruciate Ligament (ACL), through the anterolateral portal, the posterior horn and root will be easily visualized and the instruments can be smoothly brought in through the anteromedial portal (Figure 3). The pattern and extent of tears can be completely visualized (Figure 4). This helps to forestall any unwanted meniscal resection which is very common in this area.

Finally, the lateral compartment is inspected in the usual figure-offour position, as routinely performed to conclude the knee arthroscopy.

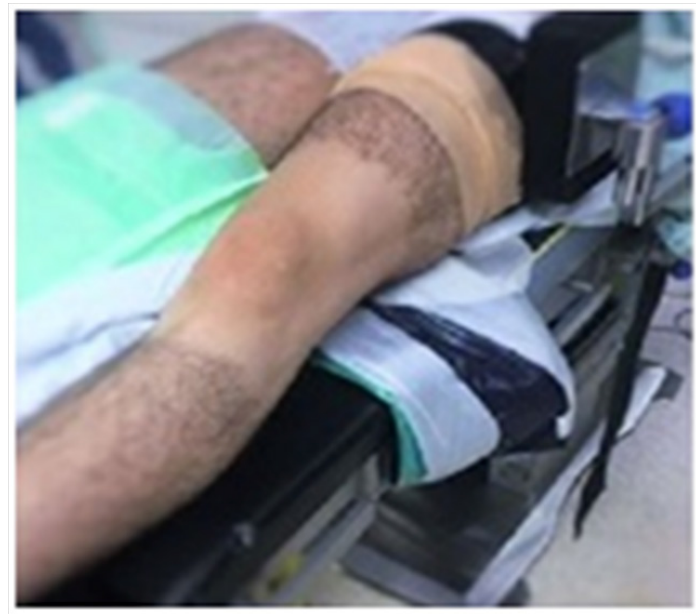

Figure I Operating table setting.

Notice the bedside padding for protection of the knee while being under varus stress.

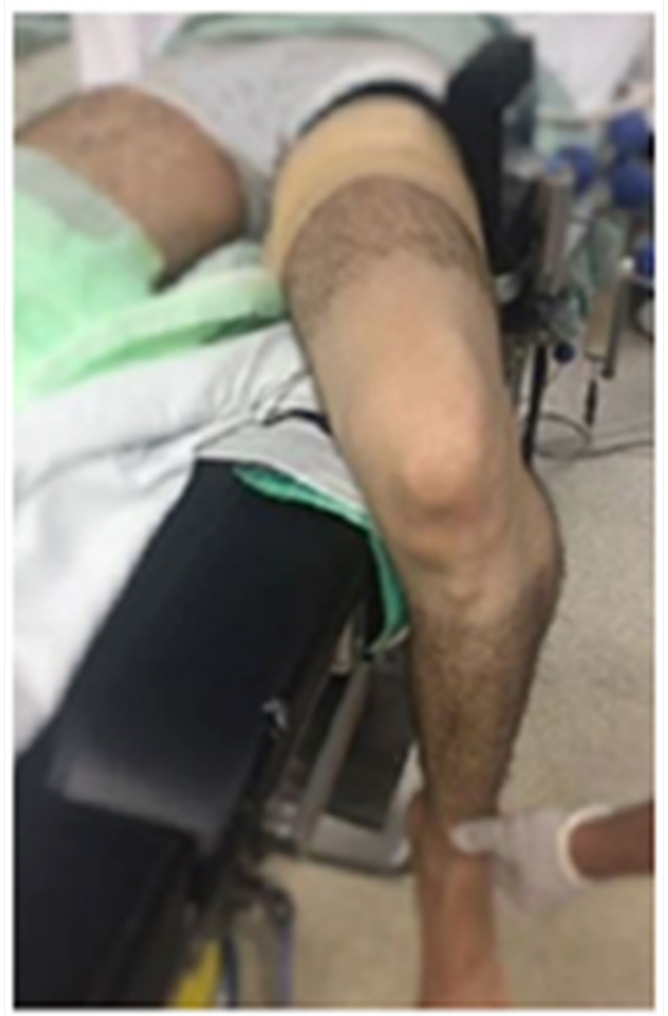

Figure 2 Pre-operative demonstration.

Varus stress is performed against a well-padded side of a regular operating table.

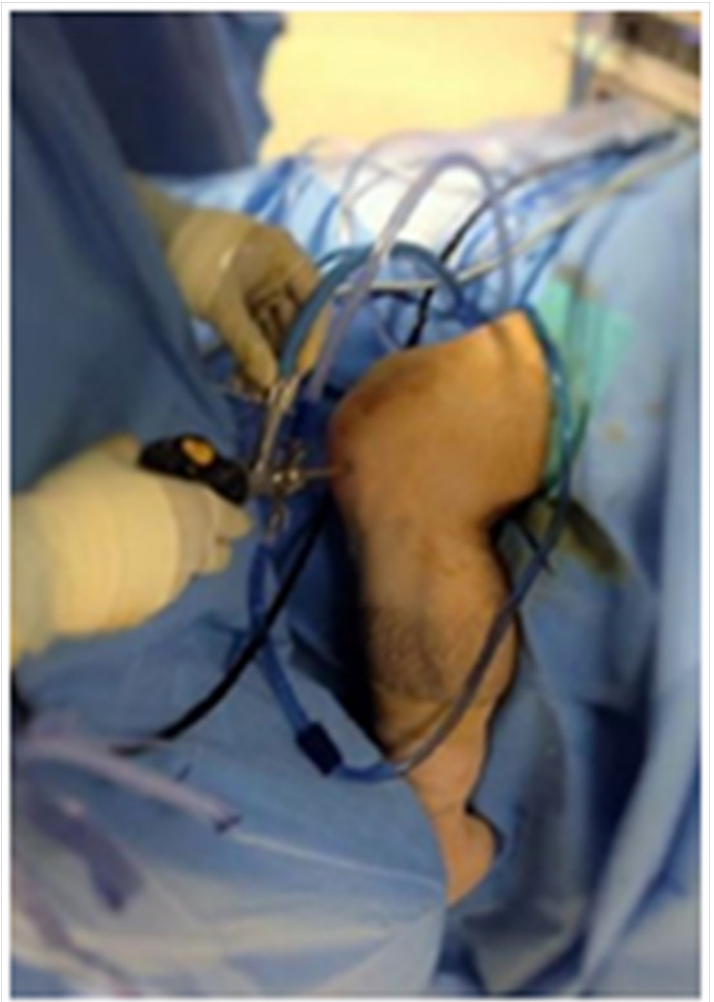

Figure 3 Intra-operative demonstration of varus stress maneuver.

Varus stress is performed by the surgeon himself, while the leg is hanging by the table in 90 degrees of flexion, with the arthroscope introduced from the anterolateral portal.

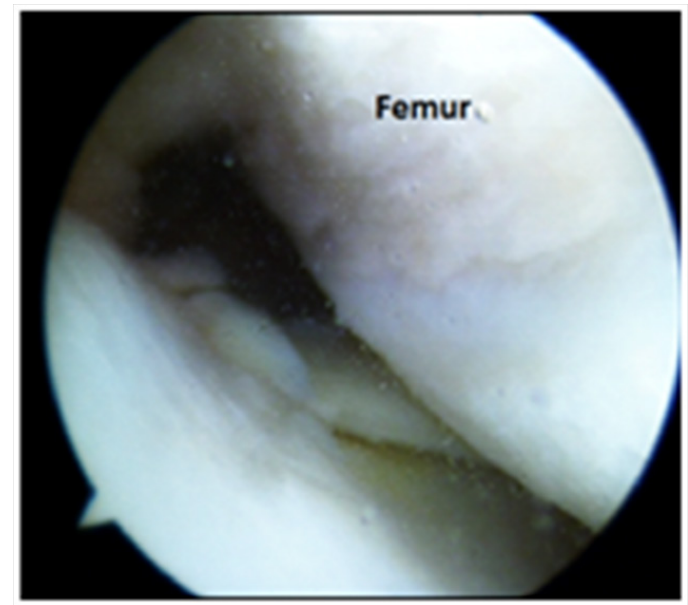

Figure 4 Arthroscopic image of left knee.

Demonstrating the ability to clearly visualize and reach the posteromedial corner.

\section{Discussion}

An absolute prerequisite for any successful arthroscopic procedure is an exact exposure of the lesion and its extent. However, due to the inherent difficulty in exposing the remote posterior horn of medial meniscus, many lesions are missed, not only by the novice but also in experienced hands. The tight posteromedial corner can make the 
passage of instruments very difficult leading to damage of intraarticular cartilage and healthy surrounding tissues. Blind cuts in this area with basket forceps can, in many cases, lead to unnecessary excision of a precious part of the medial meniscus. ${ }^{12-14}$

Our technique opens out the posterior aspect of the medial tibiofemoral compartment; with the leg hanging by the side of the operating bed, and varus stress performed by the surgeon himself. Passage of instruments is smoothly done through the intercondylar notch; hence, iatrogenic cartilage damage is avoided.

Root lesions, which are usually missed in the conventional method, can be detected using this varus stress technique. Moreover, precise delineation of the extent of bucket-handle meniscal tears is feasible using this method, and at times, the anteriorly detached buckethandle tears which flip posteriorly can be accessed and tackled. This technique, also, aids in clearly visualizing the posterior meniscosynovial attachment, when a surgeon wants to ensure the adequacy of all-inside posterior horn meniscal repair. This technique is easy, and does not require any additional portals, special instrument, legholders, or even assistants.

\section{Acknowledgments}

None.

\section{Conflicts of interest}

None.

\section{References}

1. Lohnert J, Raunest $\mathrm{J}$ Arthroscopic meniscus resection and open meniscectomy — a comparative study. Chirurg. 1986;57(11):723-727.

2. Smigielski R, Becker R, Zdanowicz U, Ciszek B Medial meniscus anatomy-from basic science to treatment. Knee Surg Sports Traumatol Arthrosc. 2015;23(1):8-14.

3. Allaire R, Muriuki M, Gilbertson L et al. Biomechanical consequences of a tear of the posterior root of the medial meniscus. Similar to total meniscectomy. J Bone Joint Surg Am. 2008;90(9):1922-1931.
4. Fithian DC, Kelly MA, Mow VC Material properties and structurefunction relationships in the menisci. Clin Orthop Relat Res. 1990;(252):19-31.

5. Kidron $\mathrm{A}$, Thein $\mathrm{R}$ adial tears associated with cleavage tears of the medial meniscus in athletes. Arthroscopy. 2002;18(3):254-256.

6. Paksima N, Ceccarelli B, Vitols A A new technique for arthroscopic resection of a bucket handle tear. Arthroscopy 14(5): 537-539.

7. Ahn JH, Oh I Arthroscopic partial meniscectomy of a medial meniscus bucket-handle tear using the posteromedial portal. Arthroscopy. 2004;20(7):e75-e77.

8. Ahn JH, Wang JH, Yoo JC A pull out suture for transection of the posterior horn of the medial meniscus: using a posterior trans-septal portal. Knee Surg Sports Traumatol Arthrosc. 2007;15(12):1510-1513.

9. Choi NH, Son KM, Victoroff BN Arthroscopic all-inside repair for a tear of posterior root of the medial meniscus: a technical note. Knee Surg Sports Traumatol Arthrosc. 2008;16(9):891-893.

10. Seil R, Dück K, Pape D A clinical sign to detect root avulsions of the posterior horn of the medial meniscus. Knee Surg Sports Traumatol Arthrosc. 2011;19(12):2072-2075.

11. Fakioglu O, Ozsoy MH, Ozdemir HM et al. Percutaneous medial collateral ligament release in arthroscopic medial meniscectomy in tight knees. Knee Surg Sports Traumatol Arthrosc. 2013;21(7):1540-1545.

12. Agneskirchner JD, Lobenhoffer $\mathrm{P}$ Arthroscopic meniscus surgery: technical-operative methods. Unfallchirurg. 2004;107(9):795-801.

13. Binnet MS, Gurkan I, Cetin C (Arthroscopic resection of bucket-handle tears with the help of a suture punch: a simple technique to shorten operating time. Arthroscopy: The Journal of Arthroscopic \& Related Surgery 2004;16(6):665-669.

14. Bosch U Percutaneous perforation of the posteromedial capsuloligamentous structures to avoid cartilaginous damage due to arthroscopic intervention at the medial meniscal posterior horn in narrow joints. Oper Orthop Traumatol 2006;18(5-6):481-484. 